Thorax (1956), 11, 10.

\title{
A THEORY OF THE ORIGIN OF INTRALOBAR SEQUESTRATION OF LUNG
}

\author{
BY \\ R. ABBEY SMITH \\ From the Thoracic Surgical Unit, King Edward VII Memorial Chest Hospital, Warwick
}

(RECEIVED FOR PUBLICATION JULY 13, 1955)

In addition to the lung tissue formed from the ventral pharyngeal diverticulum which represents the normal lung bud, pulmonary tissue may be formed from any part of the primitive foregut, and at birth is usually found lying in the left lower region of the pleural cavity; to this condition the name of lower accessory lung or extralobar sequestration is given. Such tissue should be distinguished from the additional bud which may arise from the normally developed bronchial tree, and is commonly found as an accessory lobe attached to the right side of the trachea or right main bronchus. These accessory formations may or may not survive during development ; they may or may not reveal their origin after birth by connexions with the portion of foregut, trachea, or bronchus from which they are derived; and they may receive their blood supply from the normal pulmonary trunk, from an aberrant systemic supply, or from both sources. Some are consistently associated with other congenital intrathoracic defects, others never. It is not intended to engage in the controversy that has continued for many years on the origin of the many and various congenital abnormalities that have been reported. Too many problems would require simultaneous discussion and obscure the simple purpose of this paper, which is, by using the condition defined as intralobar sequestration of lung with an aberrant arterial supply as the thesis, to inquire into the origin of the aberrant supply, the effect of this systemic supply on the structure of the lung, and to suggest an alternative explanation for the combined abnormality based on that originally described by Pryce (1946). Further information from such a discussion would seem sufficient reason for continuing to take a conjectural approach to an important clinical condition, and, although the origin of the condition is of itself unimportant to the patient, some addition may be made to the limited information on the origin and effect of systemic arterial vessels to the lung.

Bruwer, Clagett, and McDonald (1950) pointed out that anomalous arteries to the lower lobes of lungs make the lobe which they supply an interesting laboratory for the study of the effects of raised pressure. Although these authors accepted the sequestration theory of Pryce (1946) they anticipated the present hypothesis in stating:

"There is, however, no proof that anomalies of the intrapulmonic course of the branches of the normal pulmonary artery and vein may not be causal factors."

Since the observations of Haight (1942) and the paper by Pryce, Sellors, and Blair (1947) many cases have been reported emphasizing various aspects in the management of the disease. So instructive have these publications been that the condition is now diagnosed pre-operatively in most of the case reports, and these now number about one hundred. Doubt remains, however, on the origin of the condition and its relationship to lower accessory lobe. The problem of whether the aberrant artery is the prime abnormality and capable of exerting traction on the lung bud is unsolved, and there appear to be reasons for reexamining the belief that an elastic-walled aberrant vessel carrying the systemic pressure can be distributed to lung tissue that is microscopically normal (the Type 1 artery of Pryce and others). Because of this possibility it is regrettable that such fascinating conditions as total aplasia of the pulmonary artery, functional aplasia where the artery is anatomically normal (Swyer and James, 1953), and lobar agenesis with a systemic supply to the lung are outside the scope of this paper. A concurrent discussion of these with the main topic cannot be attempted for the reason given and because of inadequate first-hand experience of such cases. Such an omission does not necessarily 
vitiate the proposed hypothesis on the origin of intralobar sequestration. These gross pulmonary artery lesions have been extensively reviewed by Findlay and Maier (1951) and Maier (1954).

\section{Theories on the Origin OF INTRALObar SEQUESTRATION}

Although much has been written about the combined abnormality of the intrapulmonary mass and the aberrant artery since Pryce's (1946) paper, it is desirable to present again the essential facts of current theories on the origin of the condition. This will be done without comment.

Pryce (1946) bases his theory that the artery is the primary abnormality on the fact that it had previously been described as entering normal lung. His own experience confirmed this observation. According to this theory, the artery during foetal development captures one or more bulbous tips of the embryonic bronchial tree. Traction is then exerted on these sequestered bronchial buds, anchored by the aberrant artery, by the normal lung shifting from its original position in the region of the septum transversum. The extent of detachment of normal lung buds by this means is considered to be associated with the stage in the development of the foetal lung at which the abnormality arises. The extent of the deficiency in the normal lung is uncertain and the absence of such deficiency is in some cases explained by the ability of the normal lung to make good the deficiency on the basis of Flint's (1906) work. The lung changes are considered to be the result of continued traction by the aberrant vessel in its embryonic capillary form.

An alternative theory discussed by Bolck (1950) depends upon the occurrence of accessory lung roots in addition to the primitive lung bud. Some of these-accessory formations atrophy through failure to acquire a blood supply ; others acquire a systemic blood supply and survive. The remainder become incorporated within the normal lung at an early stage, develop normally and leave no trace of their separate existence. Their chances of separate survival depend on the distance they keep from the developing lung. Bolck considers that as the left leaf of the diaphragm closes later than the right the chances of separate survival are greater in the region of the left diaphragm and greatest if the left diaphragm is deficient and allows the separate mass further room to remain free. He thus explains the high preponderance of left-sided lesions in lower accessory lung (extralobar sequestration) and its association with congenital diaphragmatic hernia. Should the mass be incorporated in the developing lung at a relatively late stage the condition of intralobar sequestration arises. Bolck describes a case to illustrate his hypothesis.

The possibility of the combined lesion being a bronchogenic cyst with an aberrant blood supply from the aorta has been discussed. Some authors (Tosatti and Gravel, 1951 ; Weisel, Docksey, and Glicklich, 1955) describe their cases under titles which suggest that they take the view that the bronchopulmonary abnormality is a bronchogenic cyst and the anomalous blood supply an additional defect. Cole, Alley, and Jones (1951) discuss bronchogenic cysts, aberrant vessels to the diseased area, accessory lungs (extralobar sequestration), and diaphragmatic defects as related conditions. They state that the association of these anomalies suggests a common developmental error involving all structures in the lower thoracic area, and that the reason for this is some interference with the embryological organization and that any structure in the area may suffer. Finally, a number of authors (Baar and d'Abreu, 1949; Boyden, 1955) are unable to accept the aberrant artery as the primary abnormality.

It is clear that many different conceptions of the origin of the condition exist, and that there is a widespread belief that this condition and that known as lower accessory lobe (extralobar sequestration) are related embryologically. The hypothesis being put forward in this paper precludes any relationship between the two save in exceptional circumstances. It also attempts to explain the origin of intralobar sequestration by supposing that the original developmental fault is in the peripheral branches of the primitive pulmonary arterial supply; that as a result of this defect the branches from the primitive dorsal aorta persist in their early embryonic relationship to the other source of supply, and that after birth, because of the establishment of a systemic blood pressure within the aberrant circulation, the lung changes, namely cystic degeneration and fibrosis, arise and cause the final clinical and pathological picture (Fig. 1). A simplified hypothesis would suppose that the changes in the lung parenchyma originate at the same time and by the same means as the pulmonary artery defect, but there are sufficient reasons for persisting in the alternative belief until fresh conclusive evidence makes this supposition untenable. To support the present hypothesis it is necessary to attempt to establish that intralobar sequestration and accessory lung (extralobar sequestration) do not have a common origin. If this examination of the problem indicates that 

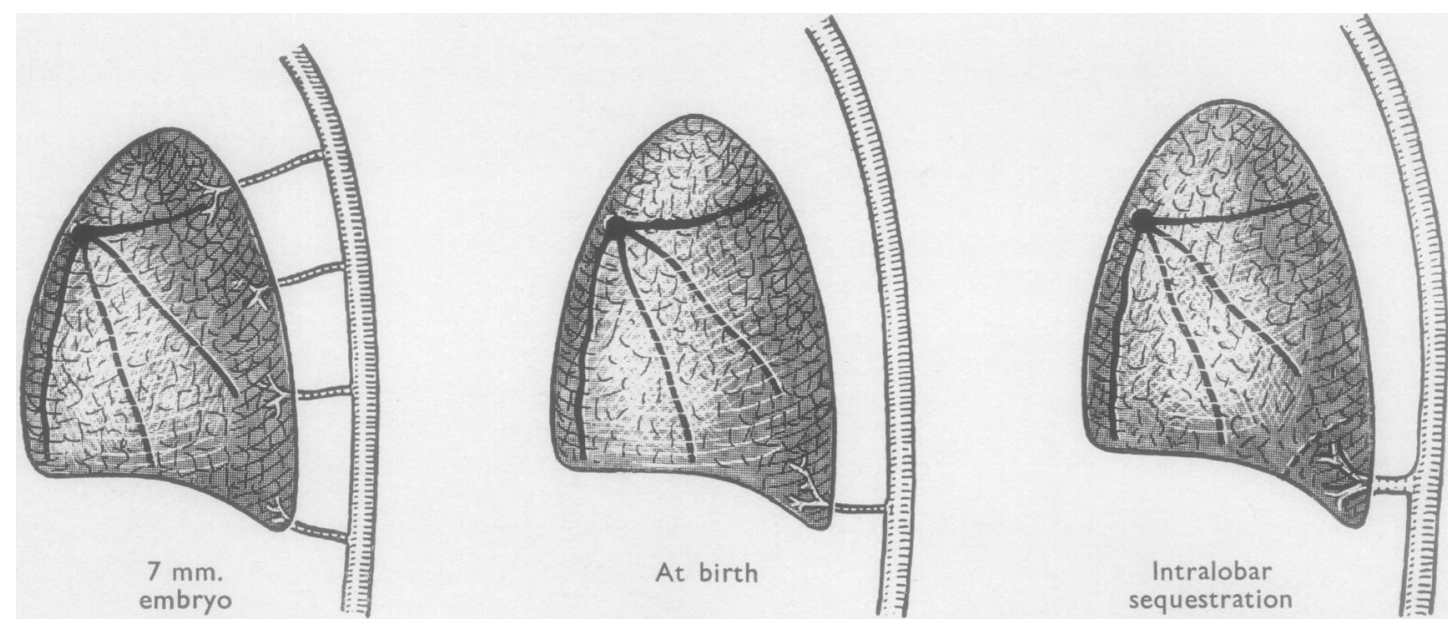

FIG. 1.-The stages in the evolution of the condition: The branches from the foetal pulmonary artery, dorsal aorta, and the morphology of the lower lobe in the $7 \mathrm{~mm}$. embryo are represented in diagrammatic form. A failure of the normal supply to reach the periphery of the posterior basal segment is represented by the broken line.

traction by the artery on the developing lung bud does in a majority of cases distract the bronchopulmonary mass from the developing lung and cause it to lie outside the confines of the normal lobe, then the theory of sequestration not only explains the origin of intralobar sequestration but those other conditions which are reported as having a common origin-notably the condition of accessory lung. It should be made clear that Pryce (1946) and Pryce and others (1947) discussed the differences between these two conditions at length, reviewed a number of alternative explanations for the formation of accessory lung, and went no further than to suggest that the origin of these two conditions demanded a common explanation. Reference may be made to the papers by Davies and Gunz (1944), Pryce and others (1947), Cole and others (1951), and Boyden (1955) for the details of the theories on the origin of accessory lung.

\section{Differences betWeEn INTRALObar SEQUeStration AND ACCESSORY LUNG}

The points of difference may be presented under seven headings.

(1) ARTERIal SupPLy.-The arterial supply from the aorta in intralobar sequestration is usually large and a significant part of the lesion. It has been described as being nearly as large as the aorta (Case 2, McDowell, Robb, and Indyk, 1955). In accessory lung the arterial pattern follows no early foetal plan, and the artery is small and insignificant. There are exceptions to this generalization. Simpson (1908) described a vessel the size of an internal carotid artery running to a small rightsided accessory lung. In this condition not only is the artery small but it is inconstant in its origin. It may arise from the aorta, from an intercostal, from phrenic branches on the diaphragm, or it may be so small that its origin has not been recorded. As far as left-sided lesions in intralobar sequestration are concerned, the aberrant vessel invariably arises from the aorta. This is associated with the differences in the manner of development of the blood supply to the two types of lung lesion. In intralobar sequestration the aberrant vessel supposedly persists because of a deficiency of the normal pulmonary supply; in accessory lung the blood supply is acquired from the nearest available supply if its development outside the lung from the earliest foetal stage is assumed. The possibility of the aberrant artery being able to exert traction on the lung bud and cause the intralobar condition to develop into the other has frequently been discussed, and a good deal of confusion exists. The aberrant vessel in intralobar sequestration is one of the ventral branches of the primitive dorsal aorta and the coeliac axis another. It is well known that during development these vessels migrate in a caudal direction, and this could afford an explanation for their supposed disrupting influence by assuming traction on the lung bud during this movement away from the lung. Such an explanation is unacceptable to embryologists. Boyden (1955) points out that the stomach in the first embryological record receives its blood supply in the cervical region, and during development through the relative caudal movement of the stomach it comes to receive its blood supply from the normal coeliac axis, some 12 segments lower, and without any "sequestration" of the stomach. This is held up as an argument against the theory 
of sequestration. In point of fact Pryce (1946) and Pryce and others (1947) never suggested any such mechanism in their original thesis. The relationship, however, between this caudal migration of vessels supplying the primitive foregut and the differences in level from which the aberrant vessel arises from the aorta is debatable, and probably the two problems are not associated. A more likely reason for the differences in level of origin of the aberrant vessel, which extend from the hilar region of the lung to the coeliac axis, is in the foetal age at which the aberrant circulation becomes established. The lower the vessel's origin from the aorta the later is the establishment of this final circulatory pattern in the foetus. The low incidence of lung changes conforming to those of intralobar sequestration in association with vessels of subdiaphragmatic origin will be mentioned later.

(2) Venous Drainage.-The venous drainage from lower accessory lung is always reported as entering the azygos system of veins: into the hemiazygos in the usual left-sided lesion. Where anomalous venous drainage of normally connected lung is concerned drainage into the azygos system of veins is extremely rare. Muir (1953) collected 89 cases of partial anomalies of venous drainage and found only two in which the drainage was into the azygos system. This suggests that normally connected lung very rarely drains to the azygos system and that its invariable occurrence in lower accessory lung indicates that such tissue has never been intrapulmonary and traction is not the reason for its extrapulmonary situation. There is only one authentic case of intralobar sequestration with drainage to the azygos vein, namely, Case 3 of Kergin (1952) which had an insignificant vein to the azygos system. Case 3 of Weisel and others (1955) had a basal lower lobe cyst on the right side draining to the azygos. This, however, was not described as a case of intralobar sequestration, as no systemic artery to the lung existed. One of Douglass's (1948) cases quoted by McDowell and others (1955) as having a vein draining into the azygos is not verified by referring to the original case report.

(3) ConNexion WITH Foregur.-The small number of cases of accessory lung with demonstrable connexions with the primitive foregut are sufficient to establish the potentiality of the primitive foregut in the formation of differentiated lung tissue. In Klebs' (1874, quoted by Gruenfeld and Gray, 1941) case the whole right lung was connected to the lower end of the oesophagus; in Scheidegger's (1936) case the connexion was through the diaphragm to the stomach; and in a case described by Gans and Potts (1951) the connexion was to the oesophagus at the level of the tracheal bifurcation. These cases of accessory lung are accepted as originating by some other developmental defect than by traction on the normal lung bud.

(4) SIDE.- In intralobar sequestration $60 \%$ of 72 cases analysed were left sided. Valle and White (1947) collected 37 cases of accessory lung and 33 of these were left sided, an incidence of $90 \%$. The predominance of left-sided lesions in lower accessory lung is important, for by the mechanism of their formation already described by Bolck (1950) their original extrapulmonary position can be presumed.

(5) POST-MORTEM Finding.-The significance of intralobar sequestration never being reported as a post-mortem finding in infancy will be discussed later. Potter (1952) had never seen the condition in 10,000 necropsies in children under the age of 1 year, and Boyd (1953) described a similar experience reporting on 6,000 necropsies from the Hospital for Sick Children in Toronto. Bodian (1955) has never identified the condition at necropsy in some 3,000 cases. In contrast to these negative findings in intralobar sequestration, in lower accessory lung the diagnosis is most frequently made as a post-mortem finding in the neonatal period. Boyd (1953) remarked on this difference between the two conditions.

(6) Anatomical Relationship. - The lung changes in intralobar sequestration are almost invariably found in the posterior basic segment of the lower lobe. The lobe is of normal configuration and only in a few cases has any sort of fissure been described separating the normal from the diseased portion. The situation of accessory lung is variable ; it may be found between the lower lobe and the diaphragm, beneath the diaphragm, in the neck, or, in three cases mentioned by Bolck (1950), in the pericardial sac. Traction by a systemic vessel could not produce this last endresult. A form of accessory lobe might be expected pedicled to the normal lobe from the point where the mass has been pulled from the lower lobe, or a partly intralobar and partly extralobar mass should be a consistent finding if traction plays a part in the origin of this condition. Such variants are not reported. The intermediate form described by Müller (1928) and quoted by Pryce (1946) is intermediate only inasmuch as the arterial root is pedicled to the parietes and the venous root to the lung. This difference in the anatomy between the arterial and venous connexions is true of all cases 
of intralobar sequestration and is not evidence of traction so long as the bronchopulmonary mass remains within the lobe, which is practically always the existing state. There are, however, four cases described in which traction by the vessel is a possibility (Case 7, Pryce and others, 1947 ; Case 5, Kergin, 1952 ; Case 4, Santy, Bérard, Galy, and Huu, 1952 ; and Case 3, Smith, 1955). Each of these right-sided lesions appeared at operation to have a fragment of lung tissue drawn into the mediastinum alongside the aberrant artery. There is also the case described by Bolck (1950) of an accessory lung with a defect in the adjoining lung corresponding to the extralobar mass in size. Because of this the author considers it certain that the accessory formation arises as a splitting off from the normal lung. Apart from these cases the evidence obtained from their anatomical relationship does not suggest a common aetiology for the two conditions.

(7) Associated Congenital Abnormality.Whereas in accessory lobe associated abnormalities are frequent, in intralobar sequestration (considering the dual abnormality as one) none have ever been described. Valle and White (1947) described 37 cases of accessory lung with diaphragmatic deficiencies in 11, and Carter and Osborn (1936) described an accessory lung with a blood supply from the coeliac axis and an associated congenital heart lesion. Hydrops foetalis and pericardial defects are two other associated abnormalities which have been reported with accessory lung. The only possible case of an associated abnormality in intralobar sequestration is one from the case records of the Massachusetts General Hospital (1943). This case had a left lower lobe cyst, a diaphragmatic hernia, and two vessels running to the lower lobe through the defect. The vessels were reported as veins, and their origin was not stated. This case was reported before the syndrome of intralobar sequestration received recognition, and it is possible that it represents the only instance of the condition being associated with an additional abnormality. In every other reported case intralobar sequestration has been the only lesion, and this suggests that its origin is some constant and localized developmental mishap which we suppose to be a failure of the pulmonary artery to the segment affected. A known but unpublished case of intralobar sequestration must be mentioned. The patient, a youth of 21 , had the typical changes in the left lower lobe with a supradiaphragmatic aortic aberrant vessel to the bronchopulmonary mass. In addition there was a cystic structure embedded in the lower oesophageal wall and reported as a teratoma. The artery coursed over the wall of the cyst before entering the lung. Such an association of abnormalities seems inexplicable by any theory other than that which defines the origin of the lesion as being due to some interference with the embryological organization in the lower thoracic area (Cole and others, 1951).

In accessory lung also an earlier and less tangible origin seems probable in most cases. Obviously if the development is traced sufficiently far back to the somite stage of the foetus, all lung tissue regardless of its situation and connexion at birth has a common origin, and whether it arises according to the accessory theory or the fraction theory becomes of less importance. If the accessory lung arises at the same time as the normal it is an accessory bud and explicable by the accessory theory; if at a later stage by a splitting off the normal bud, the explanation is by the fraction theory. These complex embryological problems are not part of the present discussion except that from this comparison of the two conditions it can be stated that the evidence for supposing that accessory lung (extralobar sequestration) and intralobar sequestration have traction by the systemic artery in common is not convincing. The differences between the two conditions are summarized in the Table.

TABLE

DIFFERENCES BETWEEN INTRALOBAR SEQUESTRATION AND LOWER ACCESSORY LUNG (EXTRALOBAR SEQUESTRATION)

\begin{tabular}{|c|c|c|}
\hline & $\begin{array}{l}\text { Intralobar } \\
\text { Sequestration }\end{array}$ & $\begin{array}{l}\text { Accessory } \\
\text { Lung }\end{array}$ \\
\hline $\begin{array}{l}\text { Arterial supply } \\
\text { Venous drainage } \ldots \\
\text { Connexion with fore- }\end{array}$ & $\begin{array}{l}\text { Large from aorta } \\
\text { Into pulmonary vein }\end{array}$ & $\begin{array}{l}\text { Small and variable } \\
\text { Into hemiazygos vein }\end{array}$ \\
\hline $\begin{array}{lll}\text { gut } & \cdots & \cdots \\
\text { Side } & \cdots & \ldots \\
\text { Diagnosed at neo- } \\
\text { natal necropsy } \\
\text { Anatomical relations }\end{array}$ & $\begin{array}{l}\text { Never } \\
60 \% \text { left } \\
\text { Never } \\
\text { Constant and intra- } \\
\text { lobar }\end{array}$ & $\begin{array}{l}\text { Sometimes } \\
90 \% \text { left } \\
\text { Frequent } \\
\text { Variable }\end{array}$ \\
\hline $\begin{array}{l}\text { Associated abnormal- } \\
\text { ity }\end{array}$ & Never & Frequent \\
\hline
\end{tabular}

The Relationship of the Pulmonary and Aberrant Circulations

Pryce (1946) has described two fundamental facts about the dual abnormality in intralobar sequestration to which it seems insufficient attention has been given by those studying this disease since publication of the original paper. These facts have a wide bearing on the problem of systemic arteries to the lung. They are (a) that the systemic artery to the bronchopulmonary mass has a wall comprised of elastic tissue, and $(b)$ that branches of the pulmonary artery to the lobe cannot be traced to the mass: in other words, that the diseased area is sequestered from its normal pulmonary arterial supply. 
The elastic nature of the wall of these arteries running to the diseased lung has not been disputed. In some case reports the microscopical appearances of the arterial wall are not given, and before their description by Pryce (1946) there was no particular interest taken in their structure. The special importance of this observation seems to be that such elastic arteries running to the lung represent a congenital anomaly which has never been reproduced after birth by any form of interference with the pulmonary artery circulation. The response to ligation of the pulmonary artery in dogs (Liebow, Hales, Bloomer, Harrison, and Lindskog, 1950) is the formation of a large number of bronchial arteries, the walls of which are of a muscular nature; in congenital heart lesions where the pulmonary flow is reduced, the oxygen requirements after birth are in part fulfilled by the growth of numerous small bronchial arteries which have muscular walls. The pulmonary artery has been ligated in an adult during an attempt to remove an inoperable lung carcinoma and, on the patient's death some months later, a considerable enlargement of muscular bronchial arteries was noted. In these, and other circumstances which need not be discussed, the pattern and distribution of the pulmonary artery to all segments of the lung is basically normal up to the time of interruption of the pulmonary flow when enlargement of arteries, the walls of which are muscular, takes place. It is logical to conclude from this that the stimulus to the development of an elastic-walled systemic artery to the lung is some disturbance during foetal life to the normal development of the pulmonary artery in its more peripheral branches, and that the systemic artery acquires its elastic coat, as does the foetal pulmonary artery, because it supplies developing lung tissue. Without going too deeply into the embryology of the blood supply to the lung, it is sufficient to say that the most likely cause of the persistence of these systemic arteries which normally take part in supplying the developing lung up to about the $8 \mathrm{~mm}$. stage (Bolck, 1950) is some breakdown in the supply from the normal pulmonary artery. There seems no more feasible explanation than this, and such a conception would satisfy those who have objected to the sequestration theory on the grounds that the aberrant artery in intralobar sequestration could not be the primary abnormality. The other observation of Pryce's, namely that the branches of the pulmonary artery could not be traced to the bronchopulmonary mass, has many times been confirmed by those who have contributed to the subject. If one considers this fact at the same time and in relation to the elastic nature of the aberrant artery, one must conclude that in intralobar sequestration the primary lesion is a failure of the normal pulmonary artery to develop to the segment involved during foetal life on the grounds that if the pulmonary artery deficiency, which is present in all cases, occurred after birth the aberrant artery should be a muscular-walled bronchial vessel.

It seems more physiological to suppose during early foetal life some minor failure of the ventral aortic supply to the lung being the cause of the persistence of the supply from the dorsal aorta than the suggestion put forward by Pryce (1946) that the component from the dorsal aorta is in competition with the supply from the ventral aorta. These two sources of supply to the developing lung before the $8 \mathrm{~mm}$. stage are complementary, and it is only by their persistence after this stage that the vessel or vessels from the dorsal aorta can be described as anomalous. They are normal and physiological before the $8 \mathrm{~mm}$. stage of development, and their atrophy depends upon the normal development of the pulmonary component. If this supply to the posterior basic segment fails it is readily compensated for by utilization of one or more of the vessels from the dorsal aorta which then become the so-called aberrant supply. It is not necessary to postulate a constant and precise stage during development for the defect to occur. If this were so the origin of the vessel from the aorta would be at a constant level in all cases. This conception of the formation of the aberrant vessel conforms to the general rule that if a foetal organ is unable to avail itself of its normal blood supply it takes up the nearest secondary supply, and if no supply is available death of the part may result. For instance, the fore limb bud of the developing newt has been transplanted during foetal life to the mid portion of the trunk, and from the nearest available supply an adequate blood supply is established to the limb with survival and a peripheral vascular arrangement resembling that of the remaining normally placed fore limb (Brandt, 1949).

The many authors who have accepted Pryce's theory have been more concerned in discussing whether these aberrant vessels represent bronchial or pulmonary arteries rather than their origin, because the aberrant artery has been accepted as the primary abnormality. It has never been established whether the vessel supplies the embryonic mesodermal element and is therefore bronchial, or the endodermal elements providing the lung alveoli and therefore pulmonary. It may supply both in some unnatural way, but it should be possible by an injection technique using a bismuth solution to establish the microscopic distribution of the vessel 
in the way that has been worked out by Cudkowicz and Armstrong (1951) for the normal bronchial vessels.

The theory presented here on the origin of the lung changes in intralobar sequestration would be more readily acceptable if it could by this means be demonstrated that the aberrant vessel supplied only the mesenchymal elements of the lung and in so doing produced fibrosis in areas normally differentiated into alveoli and cystic degeneration through a process of failure of differentiation. In their general remarks on the pathology of the disease Bruwer and others (1954) state:

"It would appear that the 'conducting' component of the involved region (that is the bronchial component) is abnormally developed and expanded into cystic structures, whereas the "functioning" (alveolar) component is undeveloped. The tissue between the expanded bronchi is largely fibrotic and contains a number of inflammatory cells depending on the amount of infection that had existed."

This is a not dissimilar proposal, and broadly speaking such a pathological state might arise from an excessive blood supply to the mesenchymal element (from the aorta) at the expense of the normal pulmonary artery. These views are open to major criticism under two heads.

(1) That the failure of the normal pulmonary artery to reach the segment involved is because this segment has already become sequestered from the normal bronchial tree by the traction of the aberrant artery, and is therefore removed from the area of distribution of the normal pulmonary artery. This would be the objection held by those who support the sequestration theory, and to answer it the evidence of there being development of lung tissue outside the normal influences which regulate the lung pattern (that is, sequestration) must be examined. Reasons for supposing that only exceptionally is intralobar sequestration a related abnormality to accessory lung (extralobar sequestration) have already been given, and these are also the reasons for supposing that traction severe enough to dislocate the bronchopulmonary mass away from the lobar boundaries does not occur. The evidence of sequestration in intralobar sequestration is more difficult to assess. Pryce (1946) suggested that the bullous cyst in one of his cases between the mass and normal lung was evidence of traction possibly due to the capillaries of the adventitious blood supply. Similar types of cyst have been recorded since. The majority of cysts in the condition have a form of bronchial epithelium and resemble the foetal alveolar ducts in structure, and they may well originate from these ducts due to a failure of normal differentiation or to their becoming blocked off and distended by their own secretions without any traction being exerted upon them. It hardly seems possible that the minor degrees of reorientation in the lower lobe which are implied in the sequestration theory could alone be the reason for the segment being deprived of a pulmonary artery supply. In the condition of simple bronchial cyst, in the common variants from normal such as an additional dorsal lobe bronchus or a cardiac lobe, the normal artery is able to furnish an adequate blood supply and, more important, it has been noted that a pulmonary artery may supply lung tissue that is actually completely dissociated from the normal lung (Baar and d'Abreu, 1949; Gans and Potts, 1951). The fact that a lesion has no demonstrable communication with a bronchus is not a reason for any failure in the normal arterial supply, and when the bronchopulmonary mass in intralobar sequestration is within the contours of an anatomically normal lobe, as the great majority are, it is hard to formulate a process by which it could be deprived of its normal blood supply if in fact this pulmonary supply is normal from an early embryonic stage. However, this valid criticism must be answered by the inherent ability of a pulmonary artery to a lobe to supply all components of the lobe in whatever relationship they stand to each other, if the artery is normal. The methods available for obtaining satisfactory evidence that the bronchopulmonary mass is sequestered from the normal bronchial tree are inconclusive. It is undoubtedly difficult to assess what lung changes can be ascribed to sequestration by the somewhat crude method of bronchography or examination of the post-operative specimen for the normality of the basal segments. A very minor disruption may be evisaged in the sequestration theory, and any such method of detecting deficiencies in the posterior basic segment bronchus, as counting the number of its divisions, would be fruitless and, as Pryce and others (1947) point out, there are other reasons than sequestration for failure to fill a $N$ basal bronchus at bronchography. In addition a N study of certain cases of intralobar sequestration which simulate cystic bronchiectasis show that normally connected lung may, when associated with an aberrant systemic circulation, undergo changes which are indistinguishable from those of intralobar sequestration except that more extensive areas are involved, and it is unnecessary to enumerate recorded instances of intralobar sequestration in lobes which are described as also showing cystic bronchiectasis in the same lobe. Thus there are these three conditions which are difficult 
to differentiate: intralobar sequestration, intralobar sequestration with bronchiectasis, and bronchiectasis in a lobe supplied by an aberrant artery. They have in common a systemic artery distributed to the diseased area. These three conditions can all be associated and explained on the present hypothesis. Degrees of sequestration were of course described by Pryce and others (1947), but it does seem necessary to assume dislocation or sequestration of bronchial buds where no evidence of this exists or simply because an aberrant artery is present. The following case is illustrative.

CASE 1.-P. H., a boy of 18 , gave a history of periodic winter cough and purulent sputum. He was otherwise healthy and without significant symptoms or signs. A plain chest radiograph (Fig. 2) showed a lesion in the left lower lobe, and a bronchogram (Fig. 3) of the left side showed a localized cystic bronchiectasis in the left lower lobe. The right lung was normal. Although the changes in the plain film seemed unusual, he was thought to have an acquired bronchiectasis and operation was advised.

At operation the left lower lobe was normal to palpation. It was immediately seen that a large aberrant vessel from the aorta ran into the dorsal segment of the lower lobe across the space between the bronchus and the inferior vein. Its origin from the aorta was unusually high. The pulmonary artery to the lower lobe was grossly reduced in size. A lower lobectomy was performed and the patient convalesced normally.

The specimen (Fig. 4) on cross section showed a large number of cystic spaces with considerable fibrosis. The most advanced changes were in the distribution of the aberrant artery, the larger branch of which ran downwards and forwards. The changes in the lung were indistinguishable from those of acquired cystic bronchiectasis on microscopical examination. The aberrant artery was $0.9 \mathrm{~cm}$. in diameter, and the elastic structure of its wall is shown (Fig. 5). The attenuated pulmonary artery was difficult to outline by "lipiodol " injection, but its distribution in the lobe was principally to the disease-free area.

By accepted standards the lobe in this case must be considered a normally connected lung free of detectable evidence of sequestration. There is no doubt, on account of the findings of a small pulmonary artery to the lobe and an elastic-walled systemic artery distributed to a fibrous cystic lesion in the lobe, that this represents an example of intralobar sequestration. The case is not dissimilar to Case 1 of Pryce and others (1947) and Case 6 of Cole and others (1951).

In a number of large series of reported cases of bronchiectasis there are references to an aberrant arterial supply. Chesterman (1951), from a total of 114 , reported two cases which showed aberrant vessels running to the lung from beneath the diaphragm, and Baar and d'Abreu (1949) reported on a case of bronchiectasis of the left lower lobe with an oesophageal diverticulum and an elasticwalled aberrant arterial supply. One of the former author's cases also had an oesophageal diverticulum. The relationship between bronchiectasis in lobes supplied by an elastic-walled aberrant arterial supply and intralobar sequestration is significant, and, if as seems probable at least some of these lesions are one and the same condition, less importance must be attached to the conception of sequestration in the development of the changes in intralobar sequestration. It is hardly necessary to state that this association of bronchiectasis with an elastic aberrant artery of similar origin and distribution to that found in intralobar sequestration is very much less frequently encountered than the association of acquired bronchiectasis, not uncommonly involving the whole lung, with a considerable enlargement of the bronchial flow probably a result of infection in the lung. The two types of vessels may as easily be differentiated at operation as by microscopical examination of their walls. The bronchial vessels are multiple, tortuous, thin walled, and bluish, and easily damaged by blunt dissection. They may be closely related to the main bronchus or enter the lung at any site, including anteriorly, as branches from the phrenic artery. The aberrant vessel of congenital origin, however, is usually single, more robust and straight running, yellowish, and in appearance resembles a piece of macaroni lying beneath the pleura, almost invariably in the region of the pulmonary ligament.

This digression leads to the second point of criticism which may be held against the viewpoint expressed here on the origin of intralobar sequestration. (2) This is that aberrant arteries have been described as running to normal lung. Pryce and others (1947) refer to case reports from the literature where the aberrant artery is reported as running to normal lung. In some they considered that the pulmonary abnormality may have been overlooked, but they concluded that the artery could supply lung that was normal (the Type 1 artery). More recently Maier (1954) has stated that anomalous arteries from the aorta may enter lungs that are grossly normal. In a study of the effects on the intrapulmonary blood vessels of a systemic arterial supply to the lung, Tejada Valenzuela, Toriello, and Thomas (1954) described a case of the tetralogy of Fallot where the right lower lobe was supplied by a vessel which, from the description, appeared to be of the congenital elastic type. They made no mention of any microscopical 


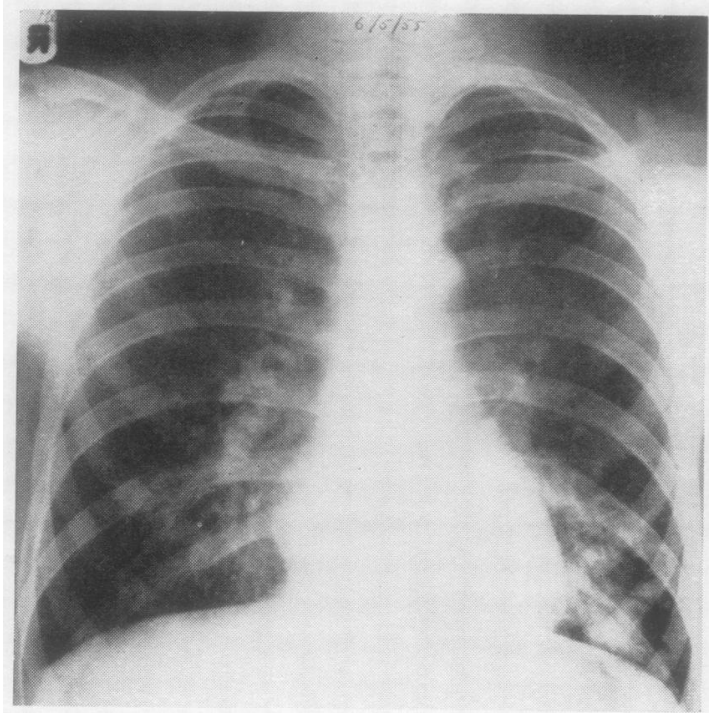

FIG.12.-Radiograph of the chest in Case 1. The appearances at the left base were thought to be due to bronchiectasis.

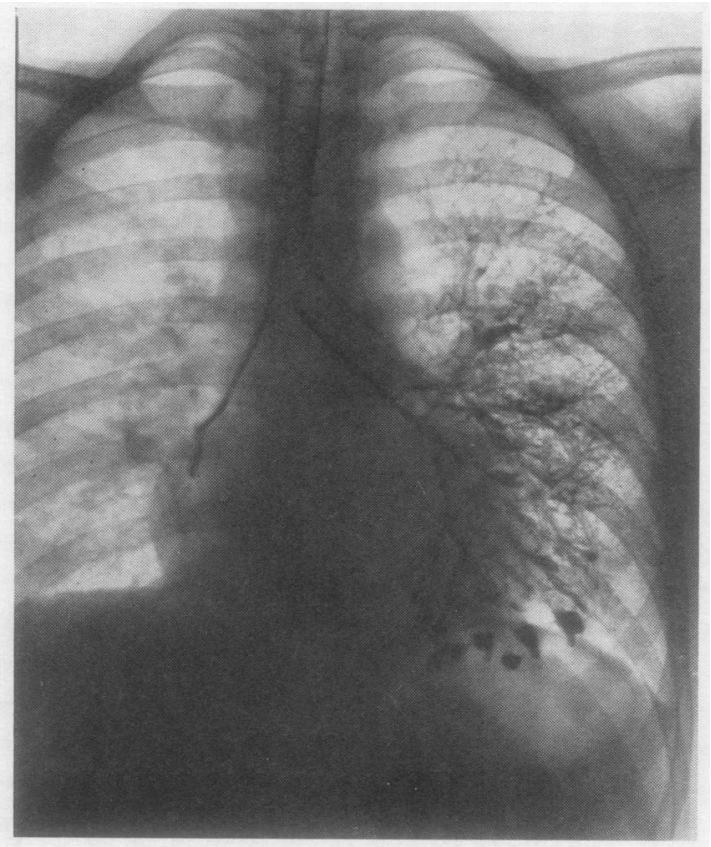

FIG. 3.-Left bronchogram (Case 1) of an apparently bronchiectatic left lower lobe.

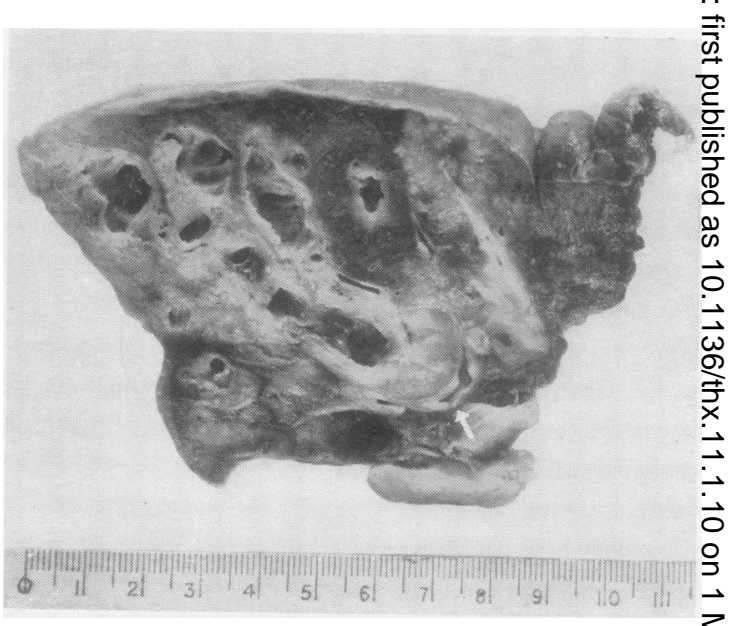

FIG. 4.-The left lower lobe in cross section from Case 1. The aberrant artery (arrowed) enters the lobe in the dorsal segment. A large descending branch running anteriorly can be seen, related $\mathcal{O}$ to the fibrotic cystic changes in the lung.

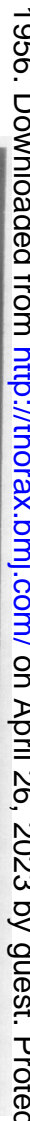

FIG. 5.-The aberrant artery in Case 1 to show elastic structure.

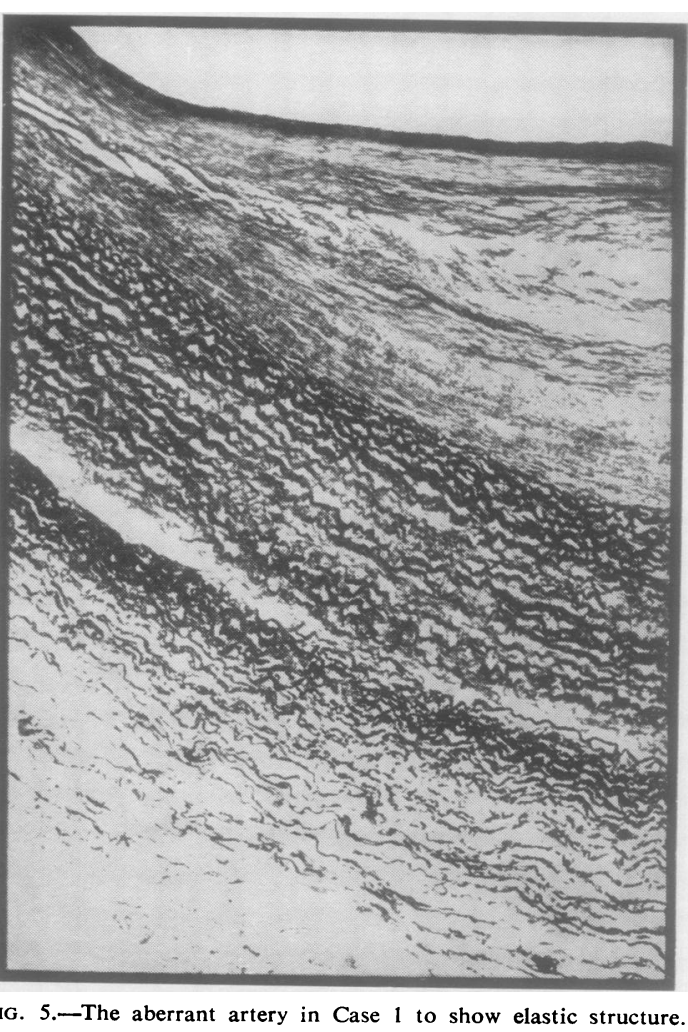

웅 
changes in the lung structure. It is therefore contrary to responsible opinion if the statement is made that the artery (Type 1) seldom enters lung that is microscopically normal. Evidence to support this contention is almost completely circumstantial. Many of the earlier reports are without microscopical studies of the lung, and palpation of the lobe at operation is insufficient evidence of normality. A number of cases of the Type 1 artery have been described in accounts of operations for congenital diaphragmatic herniae (Clay and Hanlon, 1951 ; Cole and others, 1951), and opportunity for microscopical examination of the lung has not been available. Watson (1947) described an aberrant vessel from the thoracic aorta running to a pulsatile mass in the right lower lobe. The artery was divided and the lobe not removed. Lobes showing none of the characteristic changes of intralobar sequestration but with an aberrant supply and bronchiectasis must be considered abnormal lobes. In general the opportunities for microscopy on apparently normal lobes are naturally few. The account of the pathology of the lung in the only case described by Maier (1954) in which the lung was removed is interesting. He reported little evidence of inflammation. The striking finding on microscopical examination was the uniform fibrosis of the lung with thickening of the interalveolar septa of a type not usually seen with an inflammatory process. Maier therefore wondered whether the deranged circulation played a significant role. So rare are the opportunities for full microscopical investigation of lungs which appear normal and which have an elastic-walled aberrant vessel distributed to them that until further information is available the existence of the Type 1 artery must be accepted but with certain reservations. It is not only in the present hypothesis that the Type 1 artery is difficult to explain. In the construction of any theory on the origin of intralobar sequestration the artery to normal lung is something of an embarrassment. Pryce and others (1947) explained its presence by the growth of the vessel without disruption of lung buds during development resulting in a normal lung. The reasons for the artery behaving in this way were not defined. Any theory which traces the origin of the artery as the source of blood supply to a bronchopulmonary mass which, early in foetal life, lies outside the lung and later becomes incorporated within the lobe becomes untenable if the artery can in fact run to normal lung. As far as the hypothesis under discussion is concerned, such factors as the extent to which the aberrant and pulmonary circulations overlap or are segregated may influence the structural abnormality in the lung. The presence of infection or other factors, the length of time the lung has been submitted to the systemic pressure, and the size of the vessel entering the lung may in various ways lead to degrees of lung damage which only in their developed form are those of intralobar sequestration. In contradistinction to the artery associated with this condition, the Type 1 artery commonly arises below the diaphragm and is small in diameter. This could lead to a lesser flow through the lung and offer some explanation for the absence of gross structural changes in these cases. The length of time to which the lobe is submitted to a systemic pressure may have some association with an astonishing fact about this disease. The absence of any record of the condition being demonstrated in the first few months of life at necropsy has been referred to earlier. The information provided by this large number of post-mortem examinations indicates that the lung changes may not be present at birth, and may originate only when the pressure gradient between the two circulations is established after birth and maintained for a considerable period of life. This would also explain the late age at which this presumed congenital abnormality is first diagnosed in most cases. The aberrant artery may be relatively smaller at birth whilst it carries only the pulmonary pressure and at necropsy could easily be overlooked, as it retracts into the lung substance on being cut across. That the lung changes in their fully developed form as seen in the adult could be present and yet never have been described seems hardly credible and suggests that these changes represent a gradual metamorphosis during life. It could of course be argued that the onset of infection produces the grosser evidence of the condition in later life; but there have been sufficient case reports, and one example will be described, where it can be established that infection has not been present to modify or produce the established picture of the disease. Until neonatal necropsy reports of the condition are available finality cannot be reached on this important point.

The degree to which the two circulations are dissociated may also influence the extent of the change in the lung. A complete separation of the two would seem to lead to extensive damage, although it is difficult to reconcile this with the small number of reported cases of total aplasia of a pulmonary artery to a lobe, an aberrant artery in its place, and an apparently normal lung when palpated at operation. A case demonstrating this dissociation of the two circulations will be briefly described with reference to the vascular supply before an analysis of the hypothesis is attempted. 


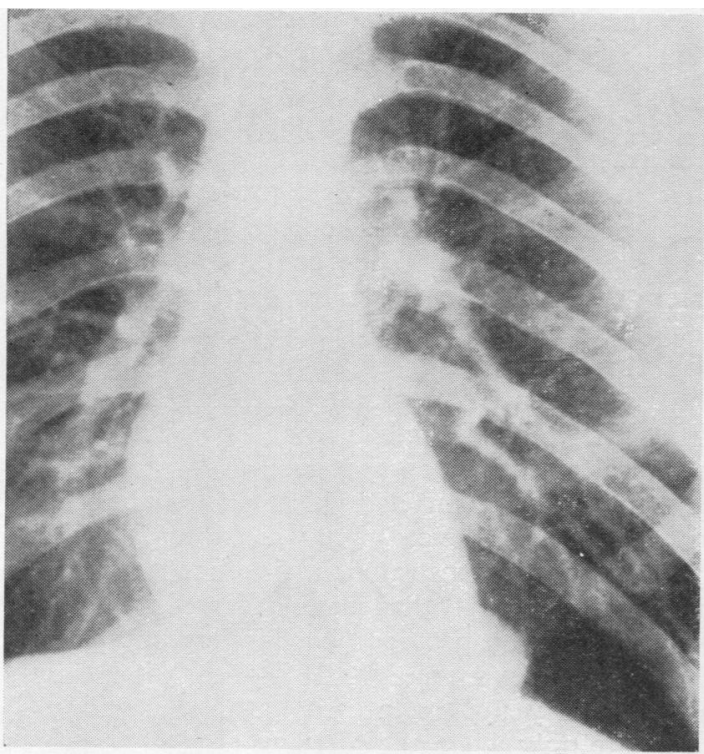

FIG. 6.-Radiograph of the chest in Case 2. The mass in the left lower lobe is obscured partly by the heart shadow and partly by the diaphragm.

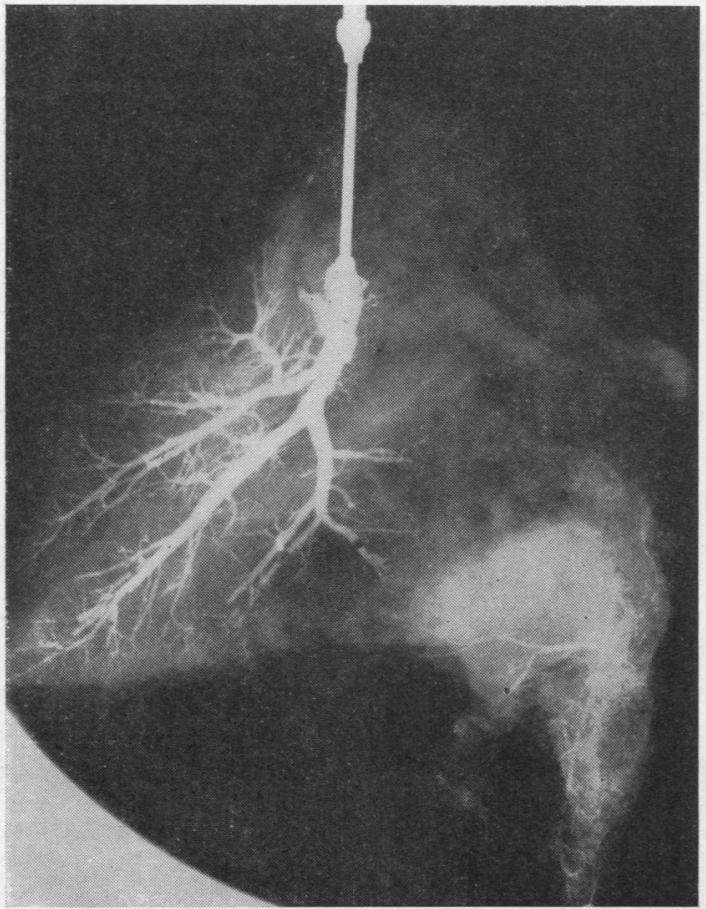

$8 \mathrm{a}$

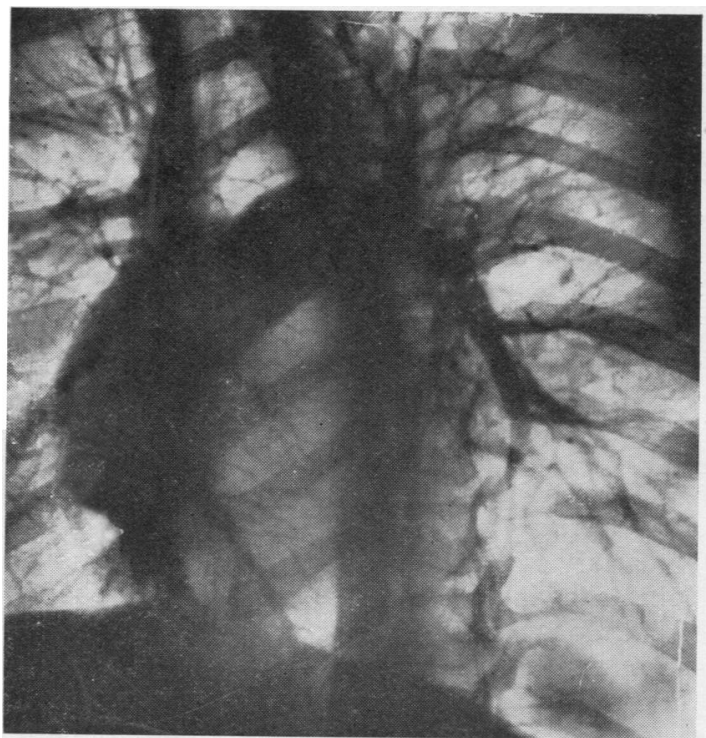

FIG. 7-Film of a 3-second angiocardiogram in the oblique positicn (Case 2). The mass in the left lower lobe and the deficiency of pulmonary vessels to this area are shown as compared with the vascular supply to the right lower lobe. Findings similar in 4 and 5 -second films.

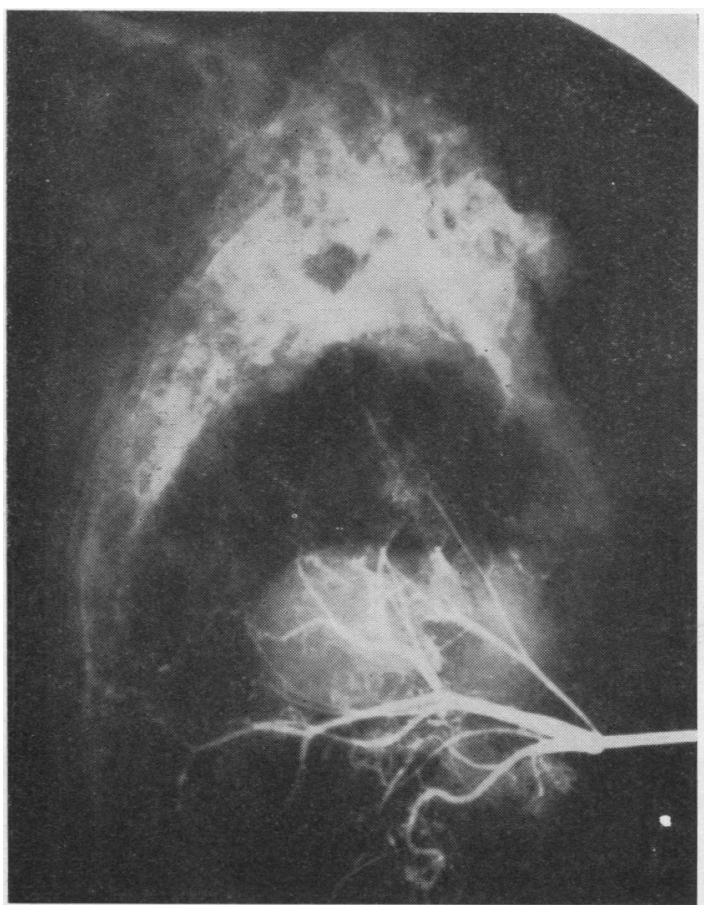

$8 b$

FiG. 8 ( $a$ and b).-Dionosil injection of pulmonary and systemic artery (Case 2). The failure of the pulmonary artery to supply the mass and the substitution of this supply by branches from the systemic supply are shown. No anastomosis between the two arterial components of the circulation exists. 
CASE 2.-S. J., a man aged 25, had never had chest symptoms or a day's illness in his life. A chest radiograph (Fig. 6) taken on entry into the Armed Forces was the reason for his being referred. All other ancillazy means of diagnosis were negative. A bronchogram was normal and a tentative diagnosis of intralobar sequestration made. To demonstrate the deficiency of the pulmonary supply to the lesion an angiocardiogram was performed (Fig. 7). An aberium vessel was not detected. At operation a cursory examination of the left lower lobe revealed no abnormality, and the lesion was at first thought to be a diaphragmatic hernia.

Examination of the pulmonary ligament showed a vessel from the aorta originating $3 \mathrm{~cm}$. caudal to the inferior pulmonary vein and entering the lower lobe. The pulmonary artery to the lower lobe was markedly reduced in size. No real abnormality of the lower

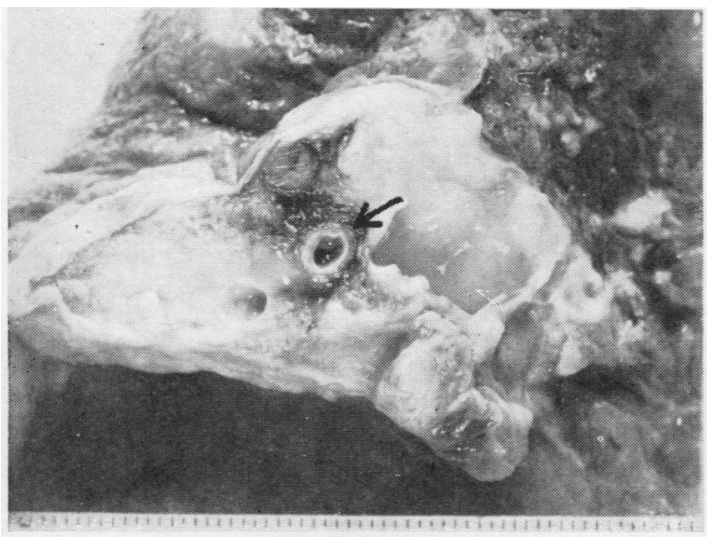

Fig. 9

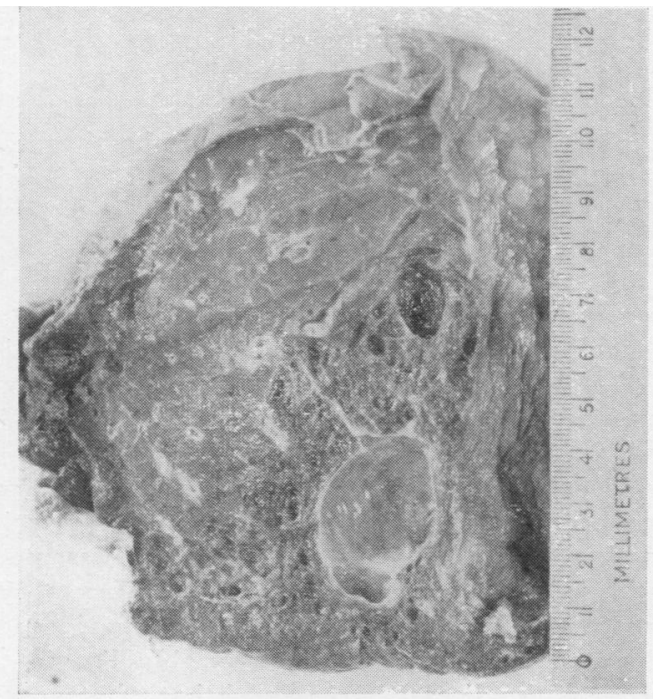

Fio. 10 lobe was noted on closer scrutiny of the lung, although the basal segment had a rubbery feel. In the absence of the obvious arterial abnormality it would have passed as normal. The basal segments were then isolated from their blood supply except for the systemic vessel. Free bleeding on releasing the clamp on the lung side of the inferior vein confirmed the return of the systemic supply to the heart by way of this vein. This end of the vein was canalized with the systemic circulation still flowing and a column of blood $17.5 \mathrm{~cm}$. above the level of the auricle was supported (with the patient in the prone position). An attempt to canalize the artery $2 \mathrm{~cm}$. from its origin was abandoned. The anomalous vessel was ligated and the removal of the basal segments completed. The patient convalesced satisfactorily.

The specimen consisted of the basal segments of the lower lobe. The aberrant artery and the pulmonary

FIG. 9. - The aberrant artery (arrowed) in Case 2 at its point of entry into the lobe. Considerable fibrosis and cyst formation around the artery is shown.

Fig. 10.-The basal segments of the left lower lobe in crcss section from Case 2. The large cyst in the posterior segment was full of mucoid secretion in life and represents the solid-looking lesion in the straight radiograph (Fig. 6).

Fig. 11.-A photograph of a whole thickness section from Case 2 at approximately the same depth as in Fig. 10. The honeycombed emphysematous appearance between the cyst and the normal lung is shown with a sharp division between the diseased area and normal lung. Normal pleura runs along the right border of the lobe.

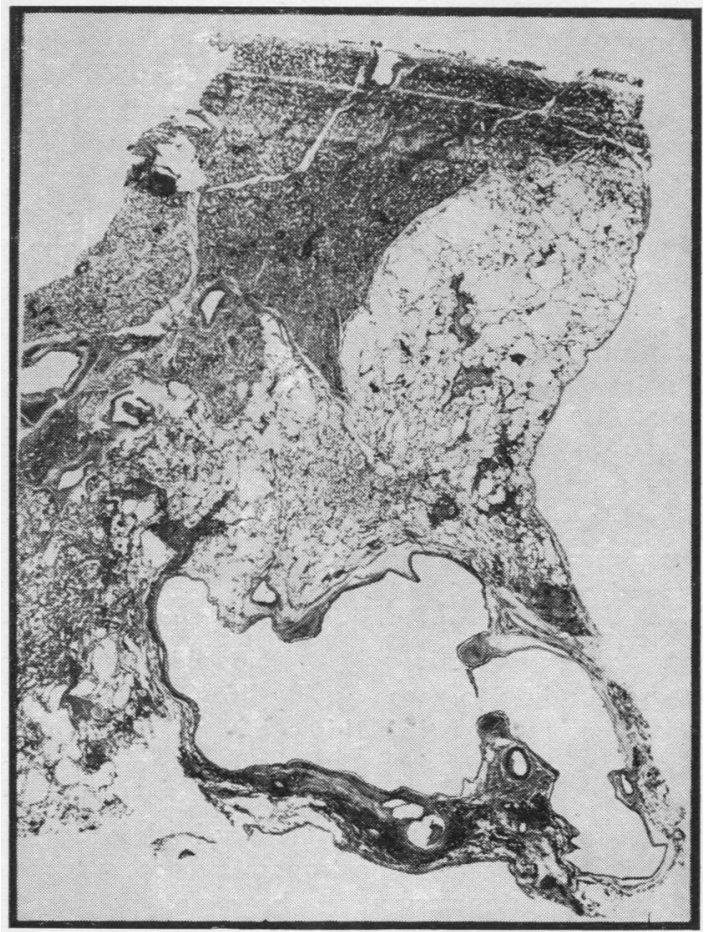

Fig. 11 
artery were injected at pressures corresponding to the systolic systemic and pulmonary pressures (Fig. 8). The aberrant artery was $0.7 \mathrm{~cm}$. in diameter, and its wall contained much elastic tissue. The disorganized lung tissue around the artery at the point of its entry into the lung is shown (Fig. 9). The artery was distributed to the bronchopulmonary mass. The mass showed the typical changes of intralobar sequestration (Fig. 10), but there was a complete absence of infection to account for the fibrosis (Fig. 11). A deficiency of the bronchial tree could not be demonstrated. It appeared that this specimen represented the condition of intralobar sequestration in a pure form.

\section{ANALysis of THE HyPOTHESIS}

Two observations in a personal series of seven cases of intralobar sequestration led to this inquiry into the origin and nature of the condition. In five of the seven cases the pulmonary artery to the lower lobe on the side of the lesion was, at operation, found to be greatly reduced in size. This has been noted previously by McDowell, Robb, Hinds, and Nicks (1951), by Cole and others (1951), by Findlay and Maier (1951), and by McDowell and others (1955). The other finding, in the specimens examined post-operatively, was that the lesions in the lung and the systemic artery were very closely related in their distribution in all cases. It can be categorically stated that this is as constant a finding in all reports of intralobar sequestration as the two previously discussed, these being that the artery has an elastic wall and that the pulmonary artery always fails to reach and be distributed to the area of the lung change.

Although causes of diminution of the pulmonary artery other than some congenital failure apparently exist, it seems too significant to ignore, particularly when so constantly associated with an anatomical deficiency of pulmonary branches to the lung lesion. The findings at angiocardiography in Case 2 (Fig. 7) not only confirm this anatomical deficiency but reveal that during life, as far as it is possible to demonstrate, the posterior segment of the lower lobe suffers an actual deprivation of normal pulmonary supply. The separation of the two circulations is adequately demonstrated, as it has been previously, by injection of the pulmonary and systemic arteries with "lipiodol" at pressures corresponding to those during life (Fig. 8). There are other reasons than this evidence for assuming that the anastomosis between the two circulations in most cases is negligible. Many observers have failed to fill the pulmonary artery by injecting the systemic artery in the post-operative specimen, and the pulmonary artery never shows any evidence of having been submitted to systemic pressure when examined microscopically for atheromatous degenerative changes in the intima. These facts suggest that one circulation has failed rather than that both sources are competing to supply the involved portion of lung. It is only necessary to consider the blood supply to another portion of the embryonic foregut - the stomach-to realize how free and widespread is the anastomosis between vessels supplying an organ if these vessels are normal. These grounds for proposing that the pulmonary artery deficiency is the principal lesion seem reasonable, and the reason for supposing that it is a congenital maldevelopment rather than an acquired extra-uterine defect has been given. It offers a satisfactory embryological explanation for the continuation of the alternative foetal supply to the lung.

The reason for the lung lesion being in the posterior basic segment is because this is the area furthest away from the main pulmonary artery, or, put in another way, the peripheral pulmonary artery branches have the longest course to this area. The flexed position of the foetus in utero causes a relative increase in this distance. The slight preponderance of left-sided lesions can be explained by the distance being less on the right side due to the elevation of the right diaphragm by the large liver of the foetus which takes a greater proportion of the available space than in the newborn. In the rare instances of bilateral systemic supply to the lower lobes a central pulmonary artery weakness is envisaged, producing a bilateral alternative supply.

The other observation which started this investigation was that the lung changes are intimately related to the distribution of the artery. This can be confirmed by referring to the reports of all recorded specimens of intralobar sequestration. It is of course an integral part of the theory of sequestration put forward by Pryce (1946), who related the lung change to traction by the aberrant artery. There are other indications from examination of resected lobes that this lung change may be a result of some other process than traction, if the embryological improbability of such traction is disregarded for the moment. These have been considered in a previous publication (Smith, 1955) and need not be described in detail. They consisted of the fact that a simple bronchogenic cyst may occur in a lobe without any factor producing traction, but when a systemic artery is present the pathological picture is materially different and structural disorganization of the lung is more advanced ; that the artery invariably lies in the centre of the diseased area ; and that whatever the 
level of origin of the systemic supply from the aorta, which might be supposed to produce traction in different directions, the lung change is always related to the branching of this vessel within the lobe, in a way which suggests some other effect than traction on or sequestration of lung buds. For these reasons and others related to earlier work on the association of lung changes (bronchiectasis) and an increase in the systemic supply to the lung, a suggestion was made that the final pathological lesion in intralobar sequestration was due to the effect of the systemic supply in the portion of the lobe to which the vessel was distributed. As a theoretical explanation for the lung damage, Pryce's conception that the changes result from damage by vascular disruption, and the present hypothesis that the lung changes arise from the establishment of a systemic supply to the involved lung, are virtually as one, but if the latter is correct important practical issues are involved in relation to an aberrant systemic supply to the lung.

If traction is not accepted, it remains to explain the means by which the systemic supply produces the lung damage. This is a highly speculative matter. Proof of this point would entail many years of experimental work on the structure of the lung and the arterial wall in infancy, the actual pressures during life in the sequestered systemic circulation, the effects of an artificially induced systemic circulation to the lung through channels other than the pulmonary artery, and observations on the changes produced by obliteration of the foetal pulmonary circulation in a suitable experimental creature. None of these is impossible, but the findings might well be inconclusive. Three possible explanations for the production of the lung change by the artery exist. The first has already been mentioned; that because the artery carries oxygenated blood and because of its aortic origin it may cause hypertrophy of the mesenchymal elements of the lung at the expense of the alveolar component. Another depends upon the findings of Cudkowicz and Armstrong (1953) in cases of pulmonary emphysema associated with occlusion of the pulmonary artery and an aberrant systemic (bronchial) supply to the lung. In these cases the authors found degeneration of the lung and fibrosis in the area supplied by the systemic supply. Full details are presented in the original paper (Cudkowicz and Armstrong, 1953). Finally an aspect of pulmonary development which may be relevant must be considered. It is well known that by birth the maximum number of bronchial divisions have not been attained. During foetal life some 18 generations of bronchi are formed, and additional bronchial budding continues after birth through childhood up to a further seven generations (Hamilton, Boyd, and Mossman, 1952). These buds under normal circumstances develop, through a number of unknown influences responsible for their differentiation, into normal lung tissue. Up to the time of birth the origin of the blood supply to the lung seems immaterial, for there are a number of cases of accessory lung at birth, completely isolated from the bronchial tree, which have reproduced a fair replica of adult type alveoli although their blood supply is entirely systemic. In terms of the physiological function of the alveoli this is difficult to explain, but it is more difficult to imagine that normal differentiation can proceed after birth when the blood to the portion of the lung which is actually budding and producing new alveoli is not only at a considerably higher pressure than normal but already oxygenated. Such are the conditions when the aberrant systemic circulation is established in cases of intralobar sequestration. Obviously it is only the post-natal buds which are exposed to this systemic pressure during development, and it is not inconceivable that it is for this reason that the development of these divisions is so irregular and haphazard. This may explain the multiplicity and variation in size of the cystic spaces that occur (Fig. 11), the close association of artery and lung lesion, the peripheral d'stribution of the lesion and the "fissure" which has been described as separating the diseased from normal lung. It also makes this hypothesis in intralobar sequestration compatible with the finding that in cases of accessory lung examined at necropsy soon after birth a satisfactory reproduction of adult alveoli is present even though the blood supply to the isolated lung tissue is exclusively systemic. The post-natal divisions are fairly constant in number, but it is not permissible to conclude that the lung lesion in intralobar sequestration should therefore be constant in size, because the final extent of the lesion, if this supposition is tenable, depends primarily on the extent of the distribution of the aberrant vessel. A concept such as this could not explain the more extensive whole lobe type of lesion shown in Case 1. Whatever the explanation, it is a good deal easier to suspect that the arterial flow through the lung is the reason for the changes that occur than it is to produce the evidence. As it is so hypothetical it cannot be discussed further to any advantage. Until more data are available on the condition of intralobar sequestration there appear to be sufficient 
grounds for correlating additional facts about the disease with the hypothesis presented, and considering it as an alternative to the theory put forward by Pryce.

\section{SUMMARY}

Reasons are discussed for assuming that intralobar sequestration and lower accessory lung are not related by any mechanism which involves traction by the systemic aberrant artery. Evidence is given in favour of the view that the primary lesion in intralobar sequestration is some failure of the pulmonary artery in foetal life to supply the segment of lung involved. The reasons for this failure are not definable in precise terms. The theory offered in this paper supposes that because of this defect the systemic supply to the foetal lung persists, and as a result of the systemic pressure the lung changes arise after birth in the area of the lung to which this supply is distributed. The theory is discussed. It may not be a complete or the only explanation of the origin of this condition.

In the preparation of this paper I have had the very considerable advantage of a number of discussions with Dr. H. S. Baar and Dr. W. Brandt, and a great deal of help also in various ways from my colleagues, Mr. J. Leigh Collis, Dr. M. Israelski, Dr. M. E. MacGregor, Dr. A. P. Prior, and Mr. T. P. Whitehead. Mr. A. L. d'Abreu kindly placed all his case records at my disposal. I am indebted to Dr. Oliver Smith for the angiocardiogram and to Mr. T. L. Skuse and Mr. L. Hine, of the technical staff of the Central Pathological Laboratory, Warwick, for their continued interest in the preparation of these specimens and the photographs. Mr. G. D. Holland, A.I.B.P., of Selly Oak Hospital, produced the photomicrographs, and Fig. 1 is Mr. Pardoe's work.

\section{REFERENCES}

Baar, H. S., and d'Abreu, A. L. (1949). Brit. J. Surg., 37, 220.

Bodian M. (1955) Personal communication.

Bolck, F. (1950). Virchows Arch. path. Anat., 319, 20.

Boyd, G. (1953). Dis. Chest, 24, 162.

Boyden, E. A. (1955). Amer. J. Surg., 89, 79

Brandt, W. (1949). Lehrbuch der Embryologie, p. $306 . \quad$ Karger, Basle.

Bruwer, A., Clagett, O. T., and McDonald, J. R. (1950). J. thorac, Surg., 19, 957

(1954). Amer. J. Roentgenol., 71, 751

Carter, H., and Osborn, H. A. (1936). J. Obstet. Gynaec. Brit. Emp., 43, 1194.

Case Records of the Massachusetts General Hospital (1943). Case 29401, New Engl. J. Med., 229, 591.

Chesterman, J. T. (1951). Brit. J. Surg., 39, 263.

Clay, R. C., and Hanlon, C. R. (1951). J. thorac. Surg., 21, 57.

Cole, F. H., Alley, F. H., and Jones, R. S. (1951). Surg. Gynec. Obstet., 93, 589 .

Cudkowicz, L., and Armstrong, J. B. (1951). Thorax, 6, 343

(1953). Ibid., 8, 46.

Davies, D. V., and Gunz, F. W. (1944). J. Path. Bact., 56, 417.

Douglass, R. (1948). J. thorac. Surg., 17, 712.

Findlay, C. W. and Maier, H. C. (1951). Surgery, 29, 604.

Flint, J. M. (1906). Amer. J. Anat., 6, 1.

Gans, S. L., and Potts, W. J. (1951). J. thorac. Surg., 21, 313.

Gruenfeld, G. E., and Gray, S. H. (1941). Arch. Path. (Chicago), 31 392.

Haight, C. (1942). Discussion. J. thorac. Surg., 11, 630.

Hamilton, W. J. Boyd, J. D., and Mossman, H. W. (1952). Human Embryology, 2nd ed., p. 201. Heffer, Cambridge.

Kergin, F. G. (1952). J. thorac. Surg., 23, 55.

Klebs, E. (1874). Quoted by Gruenfeld and Gray (1941).

Liebow, A. A., Hales, M. R., Bloomer, W. E., Harrison, W., and Lindskog, G. E. (1950). Amer. J. Path., 26, 177.

Maier, H. C. (1954). J. thorac. Surg., 28, 145.

McDowell, C. Robb, D., Hinds, J. R., and Nicks, R. (1951). Brit. J. Surg., 39, 87

and Indyk, J. S. (1955). Thorax, 10, 73

Muir, A. R. (1953). Ibid., 8, 65.

Müller, H. (1928). Handbuch der speziellen pathologischen Anatomie und Histologie (Henke, F., and Lubarsch, O.), band 3, teil 1, p. 577. Springer, Berlin.

Potter, E. L. (1952). Pathology of the Fetus and the Newborn, p. 261. The Year Book Publishers Inc., Chicago.

Pryce, D. M. (1946). J. Path. Bact., 58, 457. Sell

Santy, P., Bérard, M., Galy, P., and Huu, N. (1952). J. franc. Méd. Chir, thorac, 6, i01.

Scheidegger, S. (1936). Frankfurt. Z. Path., 49, 362.

Simpson, G. C. E. (1908). J. Anat. (Lond.), 42, 221.

Simpson, G. C. E. (1908). J. Anat. (Lond.),

Swyer, P. R., and James, G. C. W. (1953). Ibid., 8, 133.

Tejada Valenzuela, C., Toriello, J., and Thomas, W. A. (1954). A.M.A. Arch. Path., 57, 51.

Tosatti, E. and Gravel, J. A. (1951). Thorax, 6, 82.

Valle, A. R., and White, M. L. (1947). Dis. Chest, 13, 63.

Watson, W.' (1947). Surgery, 22, 919.

Weisel, W., Docksey, J. W., and Glicklich, M. (1955). Amer. Rev. Tuberc., 71, 573. 